\title{
Application of SiPM arrays for the readout of a scintillator based time-of-flight detector
}

\section{A. Korzenevev, ${ }^{a}$ C. Betancourt, ${ }^{b}$ A. Blondel, ${ }^{a}$ R. Brundler, ${ }^{b}$ A. Dätwyler,${ }^{b}$ Y. Favre, ${ }^{a}$ D. Gascon, ${ }^{c}$ S. Gomez, ${ }^{c}$ P. Mermod, ${ }^{a}$ E. Noah, ${ }^{a}$ N. Serra, ${ }^{b}$ D. Sgalaberna ${ }^{a}$ and B. Storaci ${ }^{b}$}

${ }^{a}$ DPNC, University of Geneva, Geneva, Switzerland

${ }^{b}$ Physik-Institut, Universität Zürich, Zürich, Switzerland

${ }^{c}$ Institut de Ciències del Cosmos, Universitat de Barcelona, Barcelona, Spain

E-mail: alexander.korzenevecern.ch

\begin{abstract}
A feasibility study of replacing a conventional phototube with an array of SiPMs is presented. High gain, low voltage operation and insensitivity to the magnetic field make SiPMs practically useful for the light collection in a physics experiment. In addition, sensors can be assembled in a compact system which is easily scalable. In this study an array of $6 \times 6 \mathrm{~mm}^{2}$ area SiPMs was coupled to the end of a plastic scintillator counter with dimensions $150 \times 6 \times 1 \mathrm{~cm}^{3}$. The principal restriction for applications requiring an accurate evaluation of the photons arrival time is a large SiPM capacitance which results in broadening of the signal rise time. A natural solution of the problem is to read out and amplify the sensors independently, thus essentially isolating the sensor capacitances from each other. For this purpose an 8 channel SiPM anode readout ASIC (MUSIC R1) has been used. Prospects for applications in large-scale particle physics detectors of the SHIP and T2K/ND280 experiments with timing resolution below 100 ps are provided in light of the results.
\end{abstract}

PACS: $07.07 . D f, 29.40$. MC

EPS-HEP 2017, European Physical Society conference on High Energy Physics

5-12 July 2017

Venice, Italy

${ }^{*}$ Speaker. 


\section{Introduction}

A study of timing properties of a long plastic scintillator counter using an SiPM array for the readout is presented [1]. Silicon photomultipliers are widely used nowadays in high energy physics experiments. Their excellent properties such as compactness, mechanical robustness, high photon detection efficiency and low voltage operation make them particularly useful for detecting photons produced in scintillator based counters.

The fast evolution of the SiPM market opens new possibilities. Large area sensors are available nowadays on the market at a reasonable price. When assembled in a 2D array they can cover a sizable area, therefore they can be considered as a relevant replacement for traditional photomultiplier tubes (PMT). In particular, the sensors can be coupled directly to a scintillator bulk to provide a time resolution on a sub 100 ps level [1,2]. An evident advantage of an SiPM array is that it can take the form of the bar cross section, thus avoiding complex shape light-guides.

A large SiPM capacitance increases the rise time and width of the signal and worsens the time resolution. In this regard, a large monolithic sensor or many smaller sensors with common cathode and anode [2] cannot be employed. A reduction of the overall capacitance can be achieved by using an independent sensor readout to isolate the sensor capacitances from each other. Signals can be amplified and summed afterwards inside an ASIC without the drawback of adding the sensor capacitances at the input. The MUSIC chip [3] was adopted as an input stage of the acquisition system used in this study.

The test-bench used in this work can be considered as a prototype for the design of the timing detector of the SHiP experiment [4] and the ToF system proposed for the ND280/T2K upgrade [5]. Furthermore, the test-bench is used as a test ground for a recently developed ASIC MUSIC R1 [3] which was employed in a test-beam study for the first time.

\section{Test-beam setup}

A counter with dimensions $150 \mathrm{~cm} \times 6 \mathrm{~cm} \times 1 \mathrm{~cm}$ made of a EJ-200 cast plastic scintillator was exposed to a $2.5 \mathrm{GeV} / \mathrm{c}$ muon beam to study its timing characteristics as a function of position [1]. The bar was wrapped in aluminum foil. The scintillating light was read out at both ends by arrays of 8 surface-mount SiPMs S13360-6050PE (area $6 \times 6 \mathrm{~mm}^{2}$, pixel pitch $50 \mu \mathrm{m}$ ) from Hamamatsu which have been soldered in a row to a custom-made PCB. Anode outputs of SiPMs have been read out and summed by an 8-channel SiPM anode readout ASIC (MUSIC R1) based on a novel low input impedance current conveyor [3]. Cathodes of all SiPMs were connected to a common power supply. Pulse shapes were recorded by a waveform digitizer WAVECATCHER [6]. Measurements were carried out in June 2017 at the T9 beamline of the East Hall of the CERN PS.

The trigger was formed by the coincidence of signals from two beam counters installed $20 \mathrm{~cm}$ up- and downstream with respect to the bar under test. The mean value of the times registered by both beam counters was considered as a reference. A veto counter with a beam hole of $1.5 \mathrm{~cm}$ diameter was installed right after the first beam counter and was used in an anti-coincidence mode. The time resolution of the counter was studied as a function of the position of a charged-particle interaction along the $150 \mathrm{~cm}$ axis of the bar (hereinafter referred to as $x$ ). 

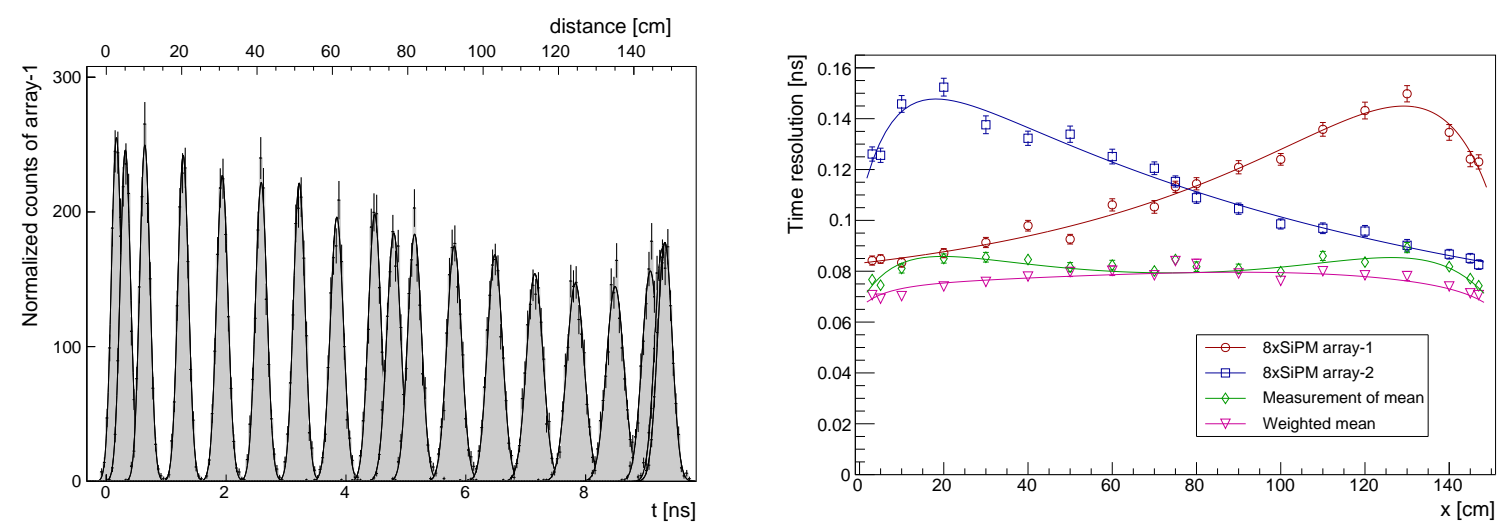

Figure 1: Left: superposition of distributions of time registered by the array-1 for different positions of an interaction point along the $x$ axis. Every distribution is approximated by a Gaussian function. Right: time resolution as measured by the SiPM arrays at both ends of the bar as a function of the interaction point along the bar.

\section{Analysis and results}

Time response of the SiPM arrays was calculated from registered waveforms in the offline analysis by applying a digital constant fraction discrimination (dCFD) technique. Examples of time spectra as measured for different positions along $x$ are shown in Fig. 1 (left). The time spectra can be reasonably approximated by Gaussian functions. For each position, the variance and the mean of the function were used to obtain the time resolution and the peak position of the distribution.

The time resolution of the counter as registered by the arrays is shown in Fig. 1 (right). It evolves from $80 \mathrm{ps}$ for the crossing point near the sensor to $150 \mathrm{ps}$ for the light propagation along the $130 \mathrm{~cm}$ distance. An improvement of the resolution is observed in case of the crossing point being at the proximity of $x=150 \mathrm{~cm}$. This could possibly be an effect of light reflected backwards. A similar effect was observed in Ref. [7]. The distribution is approximated by an analytic function (sum of two exponential functions and a constant). The resolution of the mean time and the weighted mean [7] measurements is also shown in the figure. In both cases the resolution is approximately in the region of 80 ps. However the weighted mean approach provides a visible advantage for interactions taking place in vicinity of the sensors.

\section{Application}

The technology described in this article has been proposed for the timing detector of the SHiP experiment [4] at the CERN SPS and the time-of-flight system for the upgrade of the near detector of T2K [5] at JPARC. In both cases, the transverse bar cross section of every counter is assumed to be $1 \times 6 \mathrm{~cm}^{2}$ though the bar length is a subject of the required time resolution or/and the space available for the detector. Both detectors are located in zones of strong magnetic field.

The timing detector of SHiP [4] is shown in Fig. 2 (left). The detector will be positioned downstream of the vacuum decay vessel and will have to cover a $5 \times 10 \mathrm{~m}^{2}$ area. The main purpose of the detector is a reduction of the combinatorial background (vertices made by a random muon crossing) by tagging particles belonging to a single event. The time resolution is required to be 

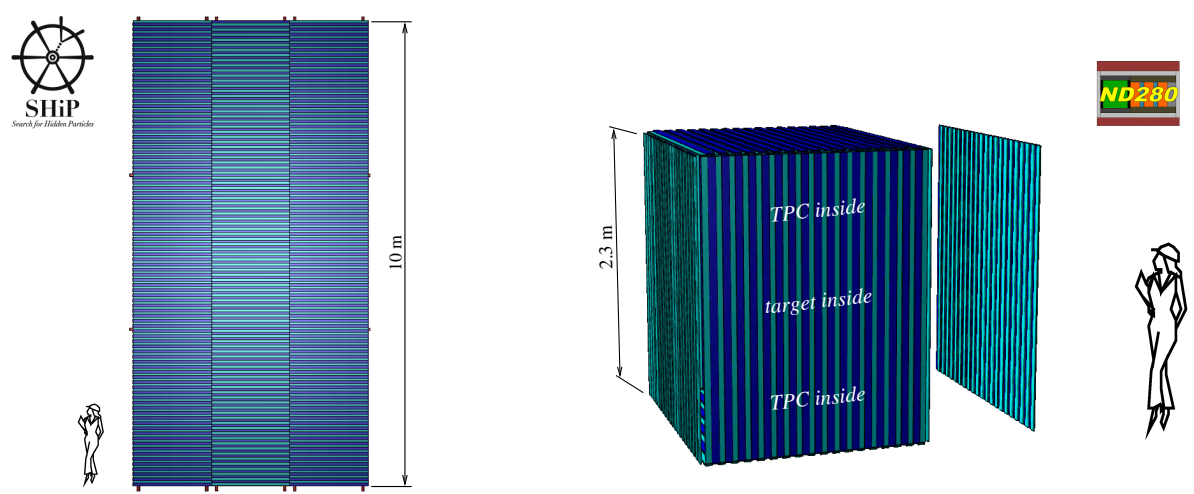

Figure 2: Schematic view of the SHiP timing detector (left) and the ToF detector proposed for the ND280/T2K upgrade (right). Both experiments are in a preparation stage.

better than 100 ps. The detector can also be used for identification of a few GeV particles. The $100 \mathrm{ps}$ resolution constraint limits the possible bar length to approximately $2 \mathrm{~m}$. Therefore an array of 3 columns and 182 rows assembled from $167 \mathrm{~cm}$ long bars is considered as a base option for the present design of the detector. Altogether, it results in 546 bars, 1092 channels and 8736 SiPMs.

The time-of-flight detector which is proposed for the upgrade of the near detector of $\mathrm{T} 2 \mathrm{~K}$ [5] is shown in Fig. 2 (right). Planes of the detector will surround the target volume and TPCs. The whole system will be located inside the UA1 magnet which will create a field of $0.2 \mathrm{~T}$. The presence of the magnetic field and a very limited space for the detector makes the use of the SiPM readout particularly advantageous. The main purpose of the detector is to determine the direction of particles (inside or outside the target) which are products of the neutrino beam interactions. Since the energy of the neutrino beam peaks at $0.6 \mathrm{GeV}$ the momentum of secondaries resides in a fewhundred $\mathrm{MeV}$ region which also makes possible to use the detector for the particle identification although the flight distance between planes is short. The detector can be assembled of 266 bars of a fixed length $2.3 \mathrm{~m}$ which provide the time resolution around $100 \mathrm{ps}$ along the bar. In total, the number of DAQ channels is 532 and the number of SiPMs is 4256 .

\section{References}

[1] C. Betancour et al., Application of large area SiPMs for the readout of a plastic scintillator based timing detector, arXiv:1709.08972, 2017

[2] M. Bonesini et al., Performance of SiPMT array readout for fast time-of-fight detectors, JINST 9 (2014) C03044

[3] S. Gomez et al., MUSIC: An 8 channel readout ASIC for SiPM arrays, Proc. SPIE 9899, 2016

[4] M. Anelli et al., A facility to Search for Hidden Particles at the CERN SPS, arXiv:1504.04956, 2015

[5] D. Sgalaberna et al., The upgrade project of the T2K near detector, proceedings of EPS-HEP 2017

[6] D. Breton et al., The WaveCatcher Family of SCA-Based 12-Bit 3.2-GS/s Fast Digitizers, HAL Id: in2p3-00995691, 2014

[7] A. Blondel et al., Study of timing characteristics of a $3 \mathrm{~m}$ long plastic scintillator counter using waveform digitizers, arXiv:1610.05667, 2016 